\title{
EXISTENCE OF LOCAL CROSS-SECTIONS IN LINEAR CARTAN G-SPACES UNDER THE ACTION OF NONCOMPACT GROUPS
}

\author{
R. A. WIJSMAN ${ }^{1}$
}

1. Introduction and summary. Let $G$ be a transformation group acting on a space $X$. If $G$ is a compact Lie group, the existence of a slice at every point of $X$ has been shown with increasing generality by Koszul [3], Montgomery and Yang [4] and Mostow [6] (definitions of various italicized terms will be given in \$2). A somewhat more restricted, but for certain purposes more useful, object is a local crosssection at a point of $X$. Its existence under some conditions was shown by Gleason [2] if $G$ is a compact Lie group. Things become much more difficult if $G$ is not assumed to be compact. It was shown by Palais [7] that if $G$ is an arbitrary Lie group, with compact isotropy group in each $x \in X$, the existence of a slice at each $x$ is equivalent to $X$ being a Cartan $G$-space.

In this paper we shall restrict $X$ to an open subset of $n$-dimensional Euclidean space, $G$ a Lie group of linear transformations of $X$ onto $X$. If, in addition, $X$ is a Cartan $G$-space, we shall show that it is possible to remove from $X$ an invariant set of Lebesgue measure 0 , such that at every point of the remaining open set $X^{0}$ there is a local crosssection. All orbits in $X^{0}$ are of maximum dimension.

A local cross-section at $x$ makes a neighborhood of the orbit of $x$ into a product space. This allows integration over the neighborhood to be carried out first "along" and then "across" the orbits. As an application in statistics we mention the problem of definition and computation of the probability density of orbits.

2. Slices and cross-sections. The terminology and notation of Palais in [7] will be followed closely. We shall define a linear $G$-space to be a couple $(X, G)$, where $X$ is an open subset of Euclidean $n$-space $E^{n}$ and $G$ is a Lie group of linear transformations of $X$ on to itself (this is slightly more restricted than the definition in [7]). When $G$ is held fixed, $X$ alone will also be called a linear $G$-space. Most definitions and lemmas in this section are equally valid for $G$-spaces that are not

Received by the editors May 25, 1965.

1 Most of the work was done at Cornell University while being on sabbatical leave from the University of Illinois. Research partially supported by NSF Grant G-21507.

The author greatly appreciates the use of facilities that were made available to him by the Department of Mathematics at Cornell University. 
necessarily linear (for definition see [7]). The image of $x \in X$ under $g \in G$ will be written $g x$. Given any basis in $E^{n}, G$ can be considered a subgroup of the group $G L(n, R)$ of all $n \times n$ real nonsingular matrices. The orbit of $x$ is $G x=\{g x: g \in G\}$. More generally, for any $S \subset X$, $G S=\{g S: g \in G\}$. The isotropy group of $x$ is $G_{x}=\{g \in G: g x=x\}$. There is a $1-1$ correspondence between $G x$ and $G / G_{x}=\left\{g G_{x}: g \in G\right\}$ $=$ the homogeneous space of left cosets of $G$ modulo $G_{x}$. If $X$ and $Y$ are any two spaces on which $G$ acts, a map $f: X \rightarrow Y$ is called equivariant if $f(g x)=g f(x)$ for all $x, g$.

A slice at $x$ is a set $S \subset X$ such that (i) $x \in S$; (ii) $G S$ is open in $X$; (iii) there exists an equivariant $\operatorname{map} f: G S \rightarrow G / G_{x}$ such that $f^{-1}\left(G_{x}\right)=S$. For future reference we state the following lemma, which is well known [7] and an immediate consequence of the equivariance of $f$ :

Lemma 1. If $S$ is a slice at $x$, then $G_{s} \subset G_{x}$ for every $s \in S$.

A local cross-section at $x$ is a slice $S$ at $x$ such that if $s$ and $g s$ are both in $S$ then $g s=s$. Thus, every orbit intersects $S$ in at most one point. A global cross-section at $x$ is a local cross-section $S$ at $x$ such that $G S=X$.

In the theory of Lie groups the notion of a local cross-section is familiar. If $G$ is a Lie group, $H$ a closed subgroup, then a local crosssection in $G / H$ is an open neighborhood $U$ of $H$ in $G / H$ together with an analytic map $\chi: U \rightarrow G$ such that $\chi(H)=e$ and $\phi \chi=$ identity map, where $\phi$ is the natural map $G \rightarrow G / H$. Then $\chi(U)$ is a local cross-section at $e$ as defined for transformation groups if we consider $G$ as an $H$-space, the action of $H$ on $G$ being right multiplication.

The lemma stated below is a specialization of Proposition 2.1.2 of Palais [7].

Lemma 2 (Palais). Let $S$ be $a$ slice at $x$ and let $\chi: U \rightarrow G$ be a local cross-section in $G / G_{x}$. Then the map $(u, s) \rightarrow \chi(u) s$ is a homeomorphism of $U \times S$ onto an open neighborhood of $S$ in $G S$.

We come now to an important characterization of a local crosssection as opposed to merely a slice.

Lemma 3. Let $S$ be a slice at $x$, then $S$ is a local cross-section at $x$ iff $G_{s}=G_{x}$ for every $s \in S$.

Proof. Let $s \in S$. As a consequence of Lemma 1 we need only show that $S$ is a local cross-section at $x$ iff $G_{x} \subset G_{s}$. First observe that $g s \in S$ iff $g \in G_{x}$, using the equivariance of $f$ defined by $S$. Then ( $S$ is a local cross-section at $x)$ iff $(g s \in S \Rightarrow g s=s)$ iff $\left(g \in G_{x} \Rightarrow g \in G_{s}\right)$.

If $S$ is a local cross-section at $x$ it follows immediately from Lemma 
3 that the map $\left(g G_{x}, s\right) \rightarrow g s$ of $G / G_{x} \times S \rightarrow G S$ is well defined and 1-1. That it is a homeomorphism follows from Lemma 2. For certain purposes it may be desirable to have $S$ a differentiable manifold. It turns out that in our linear $G$-space, if there is a local cross-section at $x$ at all, it can be chosen analytic, even "flat." We shall call a $k$-dimensional submanifold $S$ of $E^{n}$ flat if $S$ is contained in a translate of a $k$ dimensional subspace of $E^{n}$. In the following we shall restrict ourselves to flat slices and cross-sections. If $S$ is a flat local cross-section at $x$, the homeomorphism $\left(g G_{x}, s\right) \rightarrow g s$ is analytic.

Palais [7] introduced the following concepts: If $U, V$ are subsets of $X$, denote $((U, V))=\{g \in G: g U \cap V \neq \varnothing\} . V$ is called thin if $((V, V))$ has compact closure. $X$ is called a Cartan $G$-space if every $x \in X$ has a thin neighborhood. If $x$ has a thin neighborhood, $G_{x}$ is necessarily compact. For future reference we quote, below, a result of Palais, which is really a contraction of his Proposition 2.1.7 and his Lemma 2.2 in [7], stated for a linear $G$-space and slightly modified (by changing differentiability to flatness).

Lemma 4 (Palais). Let $X$ be a linear Cartan G-space, $x \in X$ and $S^{*}$ a flat submanifold of $X$ containing $x$ and invariant under $G_{x}$. Denote by $S_{x}^{*}$ and $(G x)_{x}$ the tangent spaces at $x$ to $S^{*}$ and $G x$, respectively, and suppose that $S_{x}^{*}$ is a linear complement to $(G x)_{x}$ in $E^{n}$. Then there is a neighborhood $S$ of $x$ in $S^{*}$ which is a flat slice at $x$.

The question remains under what conditions is a linear $G$-space a Cartan $G$-space. The compactness of $G_{x}$ at each $x$ is, of course, necessary, but it is not sufficient as shown by the following counter example. Let $n=4$, and write $E^{4}=E_{1} \times E_{2}$ where $E_{1}$ and $E_{2}$ are both copies of $E^{2}$. Let $X$ consist of those points of $E^{4}$ neither of whose projection on $E_{i}, i=1,2$, vanishes. Let $G$ be the additive group of reals acting on $E^{4}$ as follows: for any $-\infty<t<\infty, E_{1}$ is rotated over an angle $t$, $E_{2}$ over an angle $a t$, where $a$ is irrational. Then, for every $x \in X, G_{x}$ is trivial, but the orbit of $x$ keeps passing arbitrarily close to $x$ for arbitrarily large $t$. Thus, $X$ is not Cartan, and, moreover, there is no local cross-section at any $x$. In this example $G$ is not closed in $G L(n, R)$. If $G$ is closed and every $G_{x}$ compact it seems to be an open question whether every linear $G$-space is a Cartan $G$-space.

3. Lower-dimensional orbits. We shall use $\mu_{n}$ to denote $n$-dimensional Lebesgue measure. Let $m=\max _{x \in X} \operatorname{dim} G x$. If $x$ lies on an orbit of lower dimension, say $\operatorname{dim} G x=d<m$, then there can be no local cross-section $S$ at $x$. For, suppose the contrary, then $G_{s}=G_{x}$ for all $s \in S$, by Lemma 3 , and it follows that the orbit dimension 
would be $=d$ throughout the open set $G S$, contradicting the conclusion of

Theorem 1. Let $N=\left\{x \in X: \operatorname{dim} G x<m=\max _{y \in X} \operatorname{dim} G y\right\}$. Then $N$ is an invariant set, closed in $X$, and of $\mu_{n}$ measure 0 .

Proof. In the proof we may assume $\operatorname{dim} G>0$ and $m>0$. Let $\mathfrak{A}$ be the Lie algebra of $G$, considered as an algebra of $n \times n$ matrices. For any $x \in X$ denote by $(G x)_{x}$ the tangent space at $x$ to $G x$, then $(G x)_{x}$ $=\{A x: A \in \mathfrak{A}\}=\mathfrak{A} x$, where $x=\left(x_{1}, \cdots, x_{n}\right)^{\prime}$ is considered a column vector. Let $A_{1}, \cdots, A_{r}$ be a basis for $\mathfrak{A}$ and denote by $L(x)$ the $n \times r$ matrix whose $i$ th column is $A_{i} x$. Then $(G x)_{x}$ is spanned by the columns of $L(x)$ so that $\operatorname{dim}(G x)_{x}=\operatorname{rank} L(x)$. Now rank $L(x)<m$ for those $x$ for which all $m \times m$ determinants from $L(x)$ are 0 . Since these determinants are continuous functions of $x$, it follows that $N=\{x: \operatorname{rank} L(x)<m\}$ is closed in $X$. That $N$ is invariant is obvious from its definition. It remains to show that $\mu_{n} N=0$.

Let $y \in X$ have $\operatorname{dim} G y=m$, so that $y \neq 0$. By making a nonsingular linear transformation in $E^{n}$ we may suppose $y=(1,0, \cdots, 0)^{\prime}$. There exist $A_{1}, \cdots, A_{m} \in \mathfrak{A}$ such that $A_{1} y, \cdots, A_{m} y$ are linearly independent, i.e. the first columns of $A_{1}, \cdots, A_{m}$ are linearly independent. Let $K(x)$ be the $n \times m$ matrix whose $i$ th column is $A_{i} x$ and set $M=\{x: \operatorname{rank} K(x)<m\}$, then $N \subset M$. Premultiplying the $A_{i}$ by a nonsingular $C$ premultiplies $K(x)$ by $C$ but leaves $M$ unchanged. We shall keep the same symbols for the new matrices. Choose $C$ so that, for $i=1, \cdots, m, A_{i}$ has first column consisting of zeros except a 1 in the $i$ th row. Let $K_{1}(x)$ be the submatrix of $K(x)$ formed by the latter's first $m$ rows. Due to the form of the $A_{i}, x_{1}$ appears in $K_{1}(x)$ only in its diagonal elements, and in each of them with coefficient 1 . Let $P(x)$ be the determinant of $K_{1}(x)$, then $P(x)$ is a polynomial in $x_{1}, \cdots, x_{n}$ in which $x_{1}^{m}$ appears with coefficient 1 . Therefore, $P(x)$ is not the zero polynomial and it follows that $\mu_{n}\{x: P(x)$ $=0\}=0$. We have now $N \subset M \subset\{x: P(x)=0\}$ so that $\mu_{n} N=0$, concluding the proof of the theorem.

$G$, considered as a group of matrices, acts on $E^{n}$. By writing Theorem 1 for $E^{n}$ instead of for $X$, and observing that $X$ is open in $E^{n}$ and therefore of positive $\mu_{n}$ measure, we have the immediate

Corollary. Max $x_{x \in X} \operatorname{dim} G x=\max _{x \in E^{n}} \operatorname{dim} G x$.

As a consequence of the corollary, the maximum orbit-dimension is independent of $X$ as long as $X$ is open.

4. Existence of local cross-sections. Even if $x$ is on an orbit of maximum dimension, it is not true in general that there is a local 
cross-section at $x$, as the following example will show. Let $X$ be $E^{2}$ minus the origin and let $G$ consist of all $2 \times 2$ matrices of the form $\operatorname{diag}(c, \pm c), c>0$, then all orbits are one-dimensional. If $x$ has $x_{2} \neq 0$, $G_{x}$ is trivial, but if $x_{2}=0, G_{x}$ consists of the identity matrix and $\operatorname{diag}(1,-1)$. Using Lemma 3 it follows that no $x$ with $x_{2}=0$ can have a local cross-section (a slice $S$ at such an $x$ will intersect every $G s$, $s \in S, s \neq x$, in 2 points). This example illustrates that necessary for the existence of a local cross-section at $x$ is not only the minimality of $\operatorname{dim} G_{x}$ but also the number of components of $G_{x}$. Compare Montgomery and Zippin [5, Remark, p. 222] (for compact $G$ ). If in the example we remove the set $\left\{x: x_{2}=0\right\}$, which is of $\mu_{n}$ measure 0 , then there is a local cross-section at every point of the remainder of the space. In Theorem 2 we shall show that this can be done in general if $X$ is a linear Cartan $G$-space.

Lemma 5. If $X$ is a linear Cartan $G$-space then at each $x \in X$ there is a flat slice $S$ and a subset $S^{0}$ of $S$, relatively open in $S$ and with $\mu_{k}\left(S-S^{0}\right)=0(k=\operatorname{dim} S)$ such that at each $z \in S^{0}$ there is a flat local cross-section $S(z) \subset S^{0}$.

PRoOF. $I_{r}$ denotes the $r \times r$ identity matrix, $\Omega_{r}$ stands generically for an $r \times r$ orthogonal matrix. Take $x \in X$ arbitrarily. With the same notation as in the proof of Theorem 1 we have $(G x)_{x}=\mathfrak{A} x$. Observe that $G_{x}$ leaves $\mathfrak{A} x$ invariant. Hypotheses and conclusion of the lemma are unchanged if $E^{n}$ is subjected to a nonsingular linear transformation. Since $G_{x}$ is compact, we can choose the transformation so that $G_{x}$ becomes orthogonal [1, Theorem 1, p. 176]. Denote $\mathfrak{A} x$ by $E_{2}$ and its orthogonal complement in $E^{n}$ by $E_{1}$ and take an orthogonal basis for $E^{n}$ such that the first $k$ basis vectors span $E_{1}$. Then the matrices of $G_{x}$ have the form $\operatorname{diag}\left(\Omega_{k}, \Omega_{n-k}\right)$. Let $G_{x}^{0}$ be the subgroup of $G_{x}$ whose matrices are of the form $\operatorname{diag}\left(I_{k}, \Omega_{n-k}\right)$.

The flat manifold $S^{*}=x+E_{1}$ satisfies the conditions of Lemma 4 with $S_{x}^{*}=E_{1}$ and $(G x)_{x}=E_{2}$. By the conclusion of Lemma 4 there is at $x$ a flat slice $S \subset S^{*}$ (the existence of a slice at $x$ was, of course, already proved by Palais [7] Theorem 2.3.3). Take any $s \in S$. By Lemma 1, $G_{s} \subset G_{x}$. Let $G_{s}^{0}=G_{s} \cap G_{x}^{0}$. Take any $g \in G_{x}^{0}$ and observe $g(s-x)=s-x$, since $s-x \in E_{1}$. Combining this with $g x=x$ and using the linearity of $g$, we find $g s=s$, so $g \in G_{s}$. Therefore, $G_{x}^{0} \subset G_{s}$, and from the definition of $G_{s}^{0}$ it follows that $G_{s}^{0}=G_{x}^{0}$.

Let $H_{x}$ be the group of matrices $\Omega_{k}$ appearing in the upper left corner of the matrices $\operatorname{diag}\left(\Omega_{k}, \Omega_{n-k}\right)$ of $G_{x} . H_{x}$ may be considered to act on $E_{1}$. Define $S^{0}$ to be the relatively open subset of $S$ with the property that $s \in S^{0}$ implies that $s-x$ has a nonzero projection into 
each of the irreducible subspaces of $E_{1}$ under $H_{x}$. Then the only matrix in $H_{x}$ that leaves $s-x$ invariant is $I_{k}$. Consequently, if $s \in S^{0}$ then $G_{s}=G_{s}^{0}=G_{x}^{0}$. It follows readily from the construction that $\mu_{k}\left(S-S^{0}\right)=0$.

Take any $z \in S^{0}$. Denote $(G z)_{z}$ by $E_{2}(z)$ and its orthogonal complement by $E_{1}(z)$. Then $G_{z}=G_{x}^{0} \subset G_{x}$ implies $E_{1}(z) \subset E_{1}$. Analogous to the construction of $S$ at $x$ there is at $z$ a flat slice $S(z) \subset z+E_{1}(z) \subset z+E_{1}$ $=x+E_{1}$. Since $S^{0}$ is open in $x+E_{1}$ and contains $z$, we may choose $S(z)$ so small that $S(z) \subset S^{0}$. Now for every $s \in S^{0}, G_{s}=G_{x}^{0}=G_{z}$, and it follows then from Lemma 3 that $S(z)$ is a flat local cross-section at $z$. This concludes the proof of the lemma.

Theorem 2. If $X$ is a linear Cartan G-space there exists an invariant set $X^{0} \subset X$, open in $E^{n}$ and with $\mu_{n}\left(X-X^{0}\right)=0$, such that at every $x \in X^{0}$ there is a flat local cross-section.

Proof. To each $x \in X$ choose $S_{x}$ and $S_{x}^{0}$ according to Lemma 5 . According to Lemma 2 there is a homeomorphism of $U \times S_{x}$ onto an open neighborhood $V_{x}$ of $S_{x}$. Let $V_{x}^{0}$ be the image of $U \times S_{x}^{0}$ under this homeomorphism. Since $\mu_{k}\left(S_{x}-S_{x}^{0}\right)=0$, therefore $\mu_{n}\left[U \times\left(S_{x}-S_{x}^{0}\right)\right]=0$, and zero measure is preserved under the homeomorphism, it follows that $\mu_{n}\left(V_{x}-V_{x}^{0}\right)=0$. We have $G S_{x}=U_{o \in G} g V_{x}, G S_{x}^{0}=U_{g \in G} g V_{x}^{0}$. Since $X$ is separable, a countable union suffices: $G S_{x}=\cup_{i} g_{i} V_{x}, G S_{x}^{0}$ $=U_{i} g_{i} V_{x}^{0}$. Then $G S_{x}-G S_{x}^{0} \subset U_{i} g_{i}\left(V_{x}-V_{x}^{0}\right)$ which has $\mu_{n}$ measure 0 . Note that $G S_{x}^{0}$ is open. Furthermore, $X=\cup_{x \in X} G S_{x}$. Define $X^{0}$ $=\cup_{x \in X} G S_{x}^{0}$, then $X^{0}$ is open, invariant, and, of course, still a linear Cartan $G$-space. Again, by separability, we can cover with countable unions, and it follows as before that $\mu_{n}\left(X-X^{0}\right)=0$. Take $y \in X^{0}$ arbitrarily, then $y \in G S_{x}^{0}$ for some $x$, i.e. for some $x \in X, g \in G$ and $z \in S_{x}^{0}$ we have $y=g z$. By the conclusion of Lemma 5 there is a flat local cross-section $S(z)$ at $z$. Then $g S(z)$ is a flat local cross-section at $y$, and the proof of the theorem is completed.

Note that every point $x$ of $X^{0}$ has the following property: $x$ has a neighborhood $V$ such that $y \in V$ implies that $G_{y}$ and $G_{x}$ are conjugate. It is easy to see that in a linear Cartan $G$-space this property of a point $x$ is a necessary and sufficient condition that there be a local cross-section at $x$. Compare [2, Theorem 3.6] (for compact $G$ ). It also follows from this and Theorem 1 that every $x \in X^{0}$ has its orbit of maximum dimension.

\section{REFERENCES}

1. C. Chevalley, Theory of Lie groups. I, Princeton, N. J., 1946.

2. A. Gleason, Spaces with a compact Lie group of transformations, Proc. Amer. Math. Soc. 1 (1950), 35-43. 
3. J. L. Koszul, Sur certains groupes des transformations de Lie, Colloque de Géométrie Différentielle, Strasbourg, 1953.

4. D. Montgomery and C. T. Yang, The existence of a slice, Ann. of Math. 65 (1957), 108-116.

5. D. Montgomery and L. Zippin, Topological transformation groups, Interscience, New York, 1955.

6. G. D. Mostow, Equivariant embedding in Euclidean spaces, Ann. of Math. 65 (1957), 432-446.

7. R.S. Palais, On the existence of slices for actions of noncompact Lie groups, Ann. of Math. 73 (1961), 295-323.

UNIVERSITY OF ILLINOIS 